\title{
ETIOLOGÍA DEL CONSUMO DE ALCOHOL EN LA ADOLESCENCIA: ANÁLISIS DE DIVERSOS ENFOQUES Y TEORÍAS
}

\author{
ETIOLOGY OF ALCOHOL ABUSE IN ADOLESCENSE. \\ ANALYSIS OF DIFFERENT THEORETICAL APPROACHES
}

\author{
María Senra* \\ UNED
}

\begin{abstract}
RESUMEN
El abuso de bebidas alcohólicas entre la población adolescente es un problema ya antiguo y numerosos científicos lo han estudiado desde distintas vertientes y con enfoques diversos. En este trabajo se realiza un análisis longitudinal de la evolución del concepto de alcoholismo y su etiologia, revisando las teorías que han prevalecido en los últimos 50 años.
\end{abstract}

Palabras Clave: Alcohol abuse, alcoholism etiology, adolescence and alcohol.

\begin{abstract}
Adolescent acohol abuse is an old problem and numerous scientifics have studied it from different views. This work analized the alcoholism concept evolution and its etiology by reviewing prevalent theories during last 50 years.
\end{abstract}

Key words: Alcohol consumption, alcoholism etiology, adolescence and alcohol.

\section{Introducción}

El ser humano no nace socializado, aunque si con la potencialidad para llegar a ser un ser social. El desarrollo y canalización de esta potencialidad están condicionados por el aprendizaje. Las expectativas de un individuo, sus actitudes y su conducta se desarrollan en contacto con otros individuos, sobre todo con los miembros adultos de su entorno y con aquellos que son significativos para él.

* Maria Senra Varela es Profesora Asociada. Departamento de Orientación Educativa, Diagnóstico e Intervención Psicopedagógica (OEDIP).Facultad de Educación. UNED. Líneas de investigación: poblaciones especiales 
El conjunto de normas compartidas por un grupo social que sirven de guía en el proceso de socialización, es lo que llamamos cultura. En este proceso el individuo aprende los roles adecuados a cada situación y también las sanciones correspondientes, se dice entonces que los patrones de conducta se han institucionalizado. Pues desde el punto de vista conductual, muchas conductas bebedoras se pueden explicar como un aspecto de la cultura.

El niño imita al adulto en el consumo de alcohol igual que en cualquier otro aspecto. Tiene muy presente la imagen del adulto que bebe en cualquier acontecimiento, del adulto que se siente alegre y eufórico con la bebida, del adulto que bebe con otros adultos... y de esta forma convierte la bebida en una meta a alcanzar. De una forma subliminal el consumo de alcohol es símbolo de adultez. El consumo de alcohol se convierte así en un aprendizaje más, pero, en este caso, más tentador porque es el aprendizaje de algo que identifica con «ser mayor». Para Maddox (1970) el adolescente no inventa la idea del consumo de alcohol ¡la aprende! La aceptación y deseo del consumo de alcohol le son continuamente sugeridas por la elaborada integración del uso de alcohol en la cultura y conducta social del adulto.

La comprensión de la adolescencia tiene una particular importancia para explicar el surgimiento de la conducta bebedora o abstemia en nuestra sociedad, porque es el periodo de transición entre la infancia y la edad adulta. No es posible establecer unos límites definidos pero, en términos generales, esta etapa comienza en la pubertad, alrededor de los 12-15 años, y finaliza alrededor de los 18 , coincidiendo con la época en que el joven comienza a asumir roles de adulto. La precisión de sus límites carece de importancia, pero lo realmente importante es el hecho de que la adolescencia es una etapa de transición en la cual el joven no es «ya» un niño, pero tampoco es «todavía» un adulto. El adolescente se encuentra en un proceso de evolución hacia la adultez sin que se le permitan totalmente los roles de adulto, percibe que respecto al consumo de alcohol y tabaco, lo que es adecuado para el adulto no lo es para él aunque se le permita ocasionalmente. En tales circunstancias son lógicos los desacuerdos y tensiones generacionales que dan lugar a actitudes de rebeldía adolescente, de las cuales el consumo de alcohol puede considerarse como una muestra representativa.

El adolescente de nuestra sociedad aprende a querer ingerir alcohol del mismo modo que aprende a desear un coche, a fumar, a casarse, etc. y aprende todas estas cosas de sus padres, de su grupo de iguales, de su entorno... porque el alcohol forma parte de nuestra civilización, de nuestra cultura...

Lo aprobemos o no, el consumo de alcohol es un hábito culturalmente definido e institucionalizado de la estrategia social de muchas personas. El alcohol tiene un significado simbólico vinculado con las relaciones interpersonales y éste es el principal argumento de la mayoría de los adultos para justificar su hábito de consumo. No es sorprendente que los adolescentes puedan aportar argumentos similares para justificar su consumo de bebidas alcohólicas. Generalmente, los jóvenes consumidores aducen que beben para ser uno más en la pandilla, para celebrar ocasiones especiales o para divertirse.

Lo que el adolescente piensa y sus actitudes hacia el alcohol reflejan su integración de las tradiciones culturales adquiridas y canalizadas a través de grupos o de personas con las que se identifica, teniendo una especial influencia los padres, los amigos y los medios de comunicación.

En teoría cabría esperar que la observación de los padres es la mejor clave para entender la conducta de sus hijos adolescentes porque ellos tienen más acceso a su intimidad que ninguna otra persona o grupo social, pero la imagen de adultez que ofrecen los padres, aun- 
que es básica para los adolescentes no es el único modelo. Los compañeros y amigos de la misma edad, que pueden tener unas actitudes distintas hacia el alcohol, tienen una gran importancia en las experiencias del adolescente, especialmente en nuestra sociedad actual que atribuye un largo periodo de tiempo a la transición desde la infancia a la vida y roles de adulto. Este largo periodo de transición ha dado lugar al surgimiento de la llamada «cultura juvenil» que institucionaliza unos patrones de conducta muchas veces contrarios a los esperados por los adultos e interpretados como símbolos de hostilidad hacia ellos.

Cuando una pandilla de adolescentes consume habitualmente alcohol, se la considera un peligro por los padres de los no consumidores, pero la realidad demuestra que el adolescente puede consumir alcohol en su grupo o pandilla sin que sus padres lo sepan. Los compañeros le proporcionan el apoyo para sus primeras experiencias con la bebida, cuando ésta es considerada prematura por los adultos. El consumo de alcohol con los compañeros es, al principio, parte de un rito de iniciación.

Pero afortunadamente, también es muy frecuente que un joven se mantenga abstemio a pesar de las presiones de su grupo en el que la ingesta es habitual. Este hecho es posible porque el adolescente es capaz de encontrar motivación y refuerzo para su abstinencia en otras personas significativas para él.

En la conducta del adolescente y en sus creencias sobre el alcohol también tienen gran influencia los medios de comunicación que ofrecen al adolescente modelos de conducta estereotipados que no siempre coinciden con los que reciben de sus padres. En nuestra sociedad este problema de confrontación entre modelos de conducta contradictorios, no se reduce solamente por supuesto, al consumo de alcohol. Todos los medios de comunicación sugieren constantemente alternativas en cuanto a religión, política, vestimenta y estilo de vida en general, y no por ello se puede concluir que destruyen la moral social.

Pero no podemos descartar totalmente la influencia de los medios de comunicación en la conducta de ingesta de los adolescentes, ya que como mínimo, los anuncios publicitarios le sugieren que el consumo de alcohol en los adultos es legítimo y normal y tal sugerencia puede incitar a aquellos adolescentes en cuyo medio se le orienta hacia el consumo de alcohol.

Los padres y los iguales son, por tanto, los agentes sociales y afectivos de mayor influencia en las creencias y actitudes del adolescente hacia el consumo de alcohol. A pesar del gran impacto de los medios de comunicación, no se puede considerar su influencia como determinante en el inicio o prevalencia del consumo; en todo caso, esta influencia hay que inscribirla en el entorno del adolescente, en sus relaciones familiares y en su grupo de iguales, para relacionar y comprender su respuesta al mensaje publicitario.

Los adultos tienen legítimo derecho a esperar que los adolescentes manifiesten más responsabilidad en algunos aspectos de la que ellos mismos tienen, pero mientras la ingesta de bebidas alcohólicas sea considerado como un hábito institucionalizado de la conducta del adulto, el adolescente que está aprendiendo a ser adulto, probablemente asumirá ese hábito a pesar de todos los riesgos implícitos que le señalan los adultos. El joven, igual que el adulto, no sólo considera los riesgos del consumo de alcohol, sino también los placeres y actúa en consecuencia.

Los estudios sobre las actitudes del adolescente se han orientado hacia tres enfoques diferentes: la familia, la sociedad y la culturización, que constituyen el marco en el que el niño forma su personal visión del mundo y el pensamiento y actitudes que guiarán su vida. 
Las actitudes, creencias y conducta del joven consumidor se pueden considerar con mayor o menor grado de alarma, pero es necesario configurarlas como un problema de responsabilidad social, y para comprenderlo debemos partir de estas premisas:

1. El consumo de alcohol es un hábito culturalmente definido e institucionalizado como una experiencia social de los adultos. En este contexto social no es muy probable que se pueda persuadir al adolescente consumidor de que su conducta es inapropiada o indeseable, de que el alcohol es físicamente perjudicial y que no contribuye al logro de sus objetivos.

2. Todo lo que el adolescente aprende sobre el consumo de alcohol en diversos medios está subordinado a lo que aprende de sus padres y de sus compañeros. La publicidad por sí misma no es un factor de influencia suficiente para inducirle al consumo.

3. La educación e información sobre el consumo de alcohol debe involucrar a todos los miembros del medio ecológico, ya que su interacción juega un importante papel en el crecimiento del adolescente hacia la adultez. La educación no tiene la suficiente influencia por sí sola para persuadirle.

El Departamento de Psiquiatría de la Universidad de Columbia llevó a cabo un estudio, dirigido por Kandel (1983), de las diversas teorías acerca del consumo de alcohol en la infancia y adolescencia, y concluye que «no existe una teoría simple que explique el consumo de alcohol en la adolescencia. Sólo se puede explicar como una función de diversos factores».

Esta etimología policausal ha dado lugar a numerosos estudios con enfoques diversos tratando de dar explicación a esta enfermedad social y nos permita encontrar la panacea milagrosa que salvaguarde, al menos a los jóvenes, de esta lacra.

El propósito de este trabajo es analizar, no pormonerizadamente dada la amplitud del tema, los distintos enfoques que han dado origen a las más relevantes teorías e investigaciones en el campo del alcoholismo juvenil.

\section{La familia: ¿factor predisponente o agente causal?}

La familia es, no sólo el origen de nuestra existencia, sino las raíces sobre las que nos desarrollamos compartiendo todos los miembros una serie de características comunes, unas heredadas y otras adquiridas, como un sello de identidad familiar.

En el abuso del alcohol la familia desempeña ese doble papel, por una parte como transmisora de factores genéticos predisponentes al abuso del alcohol, y, por otra parte, como agente de socialización cuya poderosa influencia en la formación de actitudes es decisiva en la toma de decisiones respecto a la conducta consumidora o abstemia.

\section{Factores genéticos}

La tesis de que la transmisión del alcoholismo tiene sólidas bases genéticas, se apoya en amplias y extensas evidencias empíricas desarrolladas ya en 1944 por Roe. Pero como investigador pionero en este campo se considera a Jellinek, quién en 1940 inició y desarrolló estudios con familias y comprobó que el $82 \%$ de los alcohólicos pertenecían a una familia 
en la que había más de un miembro que, a su vez, eran alcohólicos. Jellinek propuso una categoría diagnóstica a partir del estudio de las familias y acunó el término «alcoholismo familiar» que posteriormente daría lugar a numerosas investigaciones tratando de buscar un factor biológico que origina o predispone al alcoholismo.

En 1960 Kaij realizó un estudio con gemelos homocigóticos y comprobó que, en el 71\% de los casos, cuando uno de los gemelos era diagnosticado como alcohólico crónico, lo era también el otro gemelo. Pero el estudio que supuso un hito y fue punto de partida de los estudios genéticos sobre la influencia de los factores biológicos en la predisposición al alcoholismo, fue realizado por Goodwin $(1973,1979)$ quien comparó, en un estudio lineal, dos grupos de niños adoptados. Un grupo estaba formado por niños cuyos padres biológicos eran alcohólicos, el otro grupo lo formaban niños de padres biológicos no alcohólicos. Todos los niños fueron adoptados por padres no alcohólicos antes de cumplir los seis meses de edad. Al alcanzar la edad adulta, Goodwin encontró que los hijos varones de padres biológicos alcohólicos desarrollaron alcoholismo con una significativa frecuencia cuatro veces superior que los hijos de padres biológicos no alcohólicos. Este hallazgo le sugirió que en los alcohólicos se puede ver implicado un factor genético que determina una diátesis al alcoholismo y que se transmite ligada al sexo masculino, ya que en las hijas de padres biológicos alcohólicos de la misma investigación, los porcentajes de alcoholismo no presentaban diferencias significativas con respecto a las hijas de padres biológicos no alcohólicos.

En un editorial del British Medical Journal (1980: 3) titulado «Alcoholismo: ¿Una enfermedad hereditaria?» se dice textualmente: «La forma en que se hereda la conducta bebedora está todavía sin establecer, pero probablemente están implicados varios genes».

A partir de los hallazgos de Goodwin son numerosas las investigaciones realizadas sobre la herencia en el alcoholismo (Cadoret, 1983; Schuckit, 1983; Vollicer, 1984) tratando de determinar la naturaleza de los factores genéticos involucrados en la transmisión del alcoholismo, así como los mecanismos de transmisión familiar de la conducta bebedora a los descendientes.

Alterman y Tarter (1986) comprobaron que el alcoholismo familiar tiene una edad de inicio temprana y tarda algún tiempo en desarrollarse. Basándose en estos datos, en un trabajo posterior (1989) comprobaron que había una relación significativa entre la conducta antisocial de los adolescentes y su situación de riesgo como hijos de alcohólicos. En 1990 Stabenau llevó a cabo una investigación para comprobar la relación significativa de los tres factores de riesgo: Conducta Antisocial, Sexo Masculino e Historia Familiar de Alcoholismo. El estudio confirmó la heterogeneidad del alcoholismo, pero los tres factores investigados correlacionaban positivamente con el alcoholismo, demostrando un alto poder predictivo y diagnóstico. Dado que los tres factores estudiados están genéticamente determinados en mayor o menor grado, Stabenau concluye que en el alcoholismo están comprometidos una heterogeneidad de fenotipos que reflejan una independencia sumativa de la varianza genética, las investigaciones biológicas requieren el aislamiento de factores neurobiológicos que identifiquen clínicamente las subformas homogéneas y los mecanismos biológicos que pueden actuar en la patogénesis de tales subformas.

Killerman (1990), a su vez, realizó un estudio, con familias de alcohólicos, sobre la variación autosómica de los factores dominantes que afectan a los movimientos involuntarios y encontró que la distonía que afecta a los alcohólicos se evidencia también en otros miem- 
bros de su familia. Lex (1991) asimismo, con una amplia investigación, establece la herencia de determinados factores biológicos que perpetúan el consumo de alcohol.

Sin embargo, el alcoholismo no se reduce a una simple y determinista cuestión biológica como comprobó Tambs (1990) al realizar un estudio con 2390 familiares de alcohólicos (esposas e hijos) encontrando que los factores genéticos y los ambientales tienen el mismo peso en la transmisión familiar del alcoholismo, puesto que, reconociendo la existencia de factores genéticos determinantes, es necesario que las condiciones ambientales sean propicias para la transmisión y desarrollo del alcoholismo. Blankfield (1991) estudió la estrecha relación entre delincuencia y alcoholismo e investigó su posible origen hereditario común, pero concluyó que su relación hay que explicarla por las leyes de la herencia como factores independientes. No obstante, la historia familiar de alcoholismo sigue siendo centro de atención de muchos investigadores y, recientemente, el Departamento de Psicología de la Universidad de Carolina del Sur llevó a cabo una investigación dirigida por Ross (2001) con el fin de establecer la influencia de la historia familiar de alcoholismo en sus hijos adolescentes, para ello realizó un estudio comparativo entre hijos de alcohólicos e hijos de bebedores sociales encontrando que los hijos de alcohólicos manifestaban una frecuencia significativamente superior de abuso del alcohol.

\section{Las actitudes en el marco familiar}

La familia, como agente de socialización por excelencia, tiene una poderosa y decisiva influencia en la formación de las actitudes infantiles. En cierto modo, actúa como el catalizador primario en la estructura actitudinal del niño. A través de la familia el niño percibe el mundo, la familia le transmite sus valores, etc. En este sentido se han desarrollado numerosos trabajos centrados en el estudio de la transmisión familiar del alcoholismo como un hábito más de la familia.

Davies (1982) realizó un estudio minucioso de los problemas familiares de alcoholismo y considera que la perpetuación del consumo en una familia se debe a la transmisión familiar del hábito, aunque sin descartar los factores biológicos, pero da vital importancia a la transmisión del hábito como cualquier otro que pueda ser peculiar, e incluso distintivo, en una familia. En la misma línea, Davies (1983) estudia las actitudes y los agentes sociales que influyen en su formación para determinar la influencia de la transmisión familiar de las actitudes hacia el alcohol. Jacob (1989) y Valleman (1990) atribuyen a la familia un papel fundamental en el desarrollo, perpetuación y transmisión del alcoholismo. Penick (1990) encontró, además, que los hijos de alcohólicos se inician en el consumo de alcohol a una edad más temprana.

Otra hipótesis a este respecto, perfiló una línea de investigación que predominó y se desarrolló a lo largo de la década de los 90, centraando su atención en el modelado parental para explicar la transmisión familiar del alcoholismo. Para Witold (1989) el padre alcohólico es un modelo a imitar y Santo Domingo (1989), en un estudio sobre la evolución de los hábitos de consumo en España, encontró una alta frecuencia de consumo excesivo de alcohol antes de los 12 años bajo la influencia familiar. Pandina (1989) afirma que la historia familiar de alcoholismo $(\mathrm{FH}+)$ puede ser un factor condicionante de disfunciones con el alcohol en la adolescencia e incluso en edades de inicio tardío. Barnes (1990) realizó un estudio con adultos alcohólicos y sus hijos, cuyos resultados revelaron que el alcoholismo paterno 
durante la etapa adolescente de los hijos, y la estructura familiar, eran factores determinantes del alcoholismo de los hijos, incluso controlando las demás variables, por lo que concluye que las influencias familiares tempranas pueden tener consecuencias a largo plazo para una conducta bebedora. A su vez Hyphantis (1991) afirma que el alcoholismo paterno es la variable con mayor peso predictivo del consumo de alcohol en la adolescencia por el fuerte modelado de la conducta de beber.

Estas conclusiones son compartidas por numerosos investigadores y han dado lugar a gran número de estudios sobre la influencia de la historia familiar de alcoholismo.

Desde otra perspectiva, Molina y sus colaboradores (1994) estudiaron la influencia familiar en la conducta de abuso del alcohol en la adolescencia, por cuanto el alcoholismo paterno determina los mecanismos temperamentales del adolescente subyacentes al abuso de alcohol y condiciona el desarrollo y desenvolvimiento social del joven haciéndole más vulnerable al abuso de alcohol (Corrao, 1995).

Las últimas investigaciones realizadas con esta orientación apuntan a la gran influencia de la familia y de hecho, el alcoholismo paterno se considera como el principal factor de riesgo para el desarrollo de dependencia alcohólica en sus hijos (Natera-Rey, 2001). Por ello los hijos de alcohólicos son en general una población de riesgo, y aquellos que abusan del alcohol comienzan su consumo a edades más tempranas (Senra, 2000) y su consumo es significativamente más intenso durante la adolescencia que el de aquellos adolescentes que no son hijos de alcohólicos (Ross \& Hill, 2001).

\section{El ambiente familiar}

El papel de agente primario de socialización por excelencia, le viene conferido a la familia porque es el primer cobijo físico y afectivo que satisface las necesidades primarias del ser humano durante un largo periodo de tiempo. No podemos olvidar que el ser más inteligente de la naturaleza es precisamente el más indefenso en sus primeros contactos con el mundo y el que más necesita una familia que le proteja y le guíe. La familia no sólo satisface sus necesidades fisiológicas, sino que le aporta estabilidad, afectividad, seguridad, etc. para que crezca sano tanto física como mentalmente. Cabe imaginar aquí la gran influencia de esas primeras vivencias en su evolución posterior. Cuando la familia falla en alguno de estos aspectos hay una carencia que puede dar lugar a desequilibrios en el desarrollo físico o psíquico del individuo.

Los estudios que relacionan la familia con el abuso de alcohol de los adolescentes, han evolucionado desde los estudios biológicos, hasta los estudios del ambiente familiar que en la actualidad generan una numerosa corriente de investigación y de aplicación de programas de prevención con mayor o menor éxito.

En una amplia investigación realizada con adolescentes de colegios madrileños (Repetto y Senra, 1997) con el fin de estudiar sus hábitos de consumo de alcohol y la relación con diversos factores, se comprobó que el ambiente familiar (estructura familiar, comunicación, cohesión, etc.) estaba relacionado con el nivel de consumo de alcohol de los adolescentes, de tal forma que se concluyó que el ambiente familiar negativo, las malas relaciones familiares y la falta de comunicación entre padres e hijos, constituyen un perfecto caldo de cultivo propicio para que el adolescente consuma bebidas alcohólicas a un nivel fuerte. La rup- 
tura de la unidad familiar debida a separaciones paternas o divorcios, colocan al adolescente en una alternativa difícil de resolver y asimilar, pero además la lucha de los cónyuges por «ganarse» el cariño de los hijos les conduce a una educación más permisiva en la que el hijo no encuentra límites ni referentes, por lo que el consumo de alcohol es un recurso para vivir nuevas experiencias. Los resultados de nuestra investigación son bien reveladores de esta realidad recientemente confirmada por Turner (2000) quien en una investigación llevada a cabo con adolescentes y sus padres y hermanos, encontró que las relaciones conflictivas entre padres o entre un hijo y sus padres, son un importante factor de riesgo para el abuso de alcohol del adolescente.

Por otra parte, la familia transmite a los hijos unos principios éticos y unas normas educativas que generalmente llevan la impronta transmitida de padres a hijos y que marcan un estilo de vida personal e intrafamiliar. Ese tipo de educación recibida de los padres es un factor importante en relación con el abuso de alcohol, ya que, en el estudio realizado con jóvenes madrileños, se comprobó que un porcentaje significativamente elevado de adolescentes que abusaban del alcohol, había recibido una educación disciplinaria y rígida en su familia. Black (1994) señala a este respecto la gran importancia de la educación basada en la comunicación padres-hijos y sugiere la importancia de programas de intervención temprana para fomentar dicha comunicación.

\section{La estructura psicológica}

El estudio de los factores psicológicos implicados en el consumo precoz de bebidas alcohólicas por niños y adolescentes, y del consumo excesivo por parte de los jóvenes y adultos, ha sido objeto de numerosas investigaciones que han seguido dos enfoques diferentes pero no excluyentes sino que en muchos puntos convergen $\mathrm{y}$, cuando menos, se pueden considerar complementarios:

\section{La personalidad infantil prealcoholómana}

Los estudios sobre la personalidad del alcohólico no son muy convincentes, desde el punto de vista etiológico, pues están realizados en alcohólicos y es muy difícil diferenciar los rasgos que fueron causa de la inclinación al alcohol de aquellos que son resultado del deterioro producido por la intoxicación etílica.

La conclusión generalizada es que no hay un sólo tipo de personalidad predispuesta al alcoholismo sino varias. El rasgo común generalizado es una falta de armonía y equilibrio entre los estratos anímicos instintivo-afectivos, por una parte, y volitivos por la otra.

El Dr. Vallejo Nájera (1981) describe dos tipos psicológicos dominantes entre los alcohólicos:

1. El tipo sensitivo: con sentimientos de inferioridad, tímidos, con marcadas dificultades para las relaciones interpersonales y carentes de habilidad para obtener afecto y amistad. 
2. El tipo opuesto tiene la misma tendencia al alcoholismo: son individuos campechanos y habladores, muy sociables, simpáticos y activos, eufóricos y con carencia de inhibiciones y autocrítica.

Los individuos del primer grupo encuentran en el alcohol la confianza en sí mismos de la que carecen y, bajo sus efectos, pueden vencer su timidez, superar su fuerte autocrítica y establecer relaciones sociales.

En el segundo tipo psicológico se encuadran aquellos individuos que están convencidos de que a ellos no les hace daño el alcohol, aun bebiendo en exceso, y no perciben su debilidad de carácter. Por mimetismo social, si pertenecen a un grupo o profesión en el que se bebe con exceso, se dejarán arrastrar sin percatarse de las señales de alarma y entrarán pasivamente en el alcoholismo.

La tesis de una personalidad prealcoholómana fue enunciada por el Profesor Alonso Fernández (1988) quien comprobó que los niños potencialmente prealcohólicos se distinguen de los demás de su misma edad y condición por mostrar un modelo de personalidad cuyas características principales son las siguientes:

1. Inmadurez afectiva en relación con los demás niños de su edad. Requieren una excesiva necesidad de amor y no soportan las frustraciones.

2. Inmadurez emocional, caracterizada por reacciones emocionales disonantes, que no corresponden con las habituales de la mayoría de los niños en situaciones semejantes.

3. Fuerte represión psicológica sobre sus instintos y sus aspiraciones, que dan como resultado niños «pusilánimes, medrosos, desesperanzados e indiferentes y aburridos.

4. Pasividad y dependencia en su conducta. Tienen tendencia a evadirse como recurso ante situaciones difíciles, y el consumo de alcohol puede formar parte de esa «huida» ante la realidad.

5. Actitud amistosa superficial. Sienten amistad hacia «los otros» pero carece de profundidad, no hay comunicación.

6. Depresión y ansiedad con un fuerte sentimiento de frustración.

Para el Profesor Alonso, los niños cuya personalidad acumula estos rasgos, constituyen una población de riesgo y pueden desarrollar cierta morbilidad alcohólica, sobre todo si alguna vez han consumido alcohol y lo han percibido como un «poder» liberador de sus timideces y creen haber encontrado un modo fácil para comunicarse o resolver una situación conflictiva; porque, la historia de muchos alcohólicos comienza con sus primeras experiencias con el alcohol, que les puede hacer creer, sin las defensas del adulto, que han encontrado la llave para sentirse poderosos, audaces y parlanchines.

León Fuentes (1990) estudió la personalidad de adolescentes consumidores habituales de alcohol y encontró como rasgos comunes: fuerte agresividad, pobreza afectiva, dificultad para establecer y desarrollar relaciones interpersonales, inadaptación social y dependencia del medio y del grupo. Power (1990) comprobó, además, que los trastornos emocionales en la infancia están estrechamente relacionados con el posterior consumo de alcohol en la adolescencia. A su vez Kornbilt (1990) en un estudio con población adolescente argentina encontró que los jóvenes que incurren en el consumo de alcohol son sujetos con una actitud 
crítica e inconformista respecto a la sociedad. Sienten «falta de sentido a su vida» y tienden a la satisfacción inmediata y al egocentrismo.

Para confirmar y definir la personalidad prealcoholómana se han llevado a cabo estudios comparativos con jóvenes de poblaciones de alto riesgo (hijos de alcohólicos) y jóvenes que abusaban del alcohol, con el fin de buscar y definir características diferenciadoras. Siguiendo esta línea, Rearden (1989) y Griffin (1990) realizaron estudios independientes y llegaron a las mismas conclusiones: Tanto los jóvenes de alto riesgo como los jóvenes que abusaban del alcohol se caracterizaban por su baja autoestima. Rearden considera que la baja autoestima es un buen predictor de la posterior conducta de ingesta, hasta el punto de afirmar que 1 de cada 10 niños con baja autoestima llegará a ser alcohólico. A similares conclusiones llegó Tweed (1991) al estudiar a poblaciones de alto riesgo, encontrando que tanto los niños como los adolescentes, hijos de alcohólicos, muestran una personalidad característica común: puntúan significativamente más alto en rasgos de ansiedad y depresión, mostrando unas características similares a los hijos de enfermos mentales. Singh (1994) también encuentra una asociación significativa entre abuso de alcohol en la adolescencia y baja autoestima, así como bajas expectativas. Black (1994) en un amplio estudio de la conducta de los adolescentes que abusan del alcohol y las drogas, concluye que estos adolescentes están involucrados generalmente en otras actividades o conductas de riesgo y tienen unas características psicológicas comunes, por lo que los rasgos psicológicos son el mejor predictor en edades tempranas de la conducta de ingesta. La baja autoestima, característica de los adolescentes hijos de alcohólicos, es más acentuada y notoria en aquellos que, además, abusan del alcohol (Ibáñez y Senra, 1999).

\section{Las variables temperamentales}

Hace ya más de medio siglo un médico y psicólogo, McDougall, advirtió que los individuos de temperamento extrovertido respondían al alcohol de forma muy diferente a la que mostraban los sujetos introvertidos. Aunque McDougall no acertó a explicar el fundamento biológico de esas diferentes respuestas, las investigaciones posteriores las confirmaron y explicaron científicamente.

Eysenck (1965), al estudiar la relación entre neurosis y alcoholismo, estableció la tesis de las variables temperamentales extroversión-introversión y neoriticismo-psicoticismo como explicación predictiva de la conducta alcohólica. Eysenck comprobó que los sujetos extrovertidos poseen un nivel habitual de excitación cortical significativamente más bajo que el de los introvertidos. Cuando una persona ingiere alcohol, tanto si es introvertida como extrovertida, experimenta un efecto depresor y su nivel de excitación cortical disminuye. La diferencia estriba en que la disminución del introvertido parte de un nivel más alto que la del extrovertido y, en consecuencia, mientras el introvertido se manifiesta más relajado, sociable y eufórico, el extrovertido, que parte de un nivel de excitación cortical más bajo, al disminuir éste con el alcohol, pierde el autocontrol.

Al descender de un nivel elevado a un nivel medio, el introvertido libera la actividad subcortical responsable del tono afectivo, de la motivación y de las emociones; se siente más eufórico y comunicativo, pero conserva una capacidad de control suficiente para regular su conducta. En el extrovertido el descenso se hace partiendo ya de un nivel de actividad 
Etiología del consumo de alcohol en la adolescencia: análisis...

cortical bajo, por lo que los efectos de la carencia de control provocada por el alcohol son más fuertes e inmediatos.

Basándose en estos hechos Eysenck afirma que la extroversión, como rasgo temperamental predominante, es un factor de riesgo significativo de la conducta alcohólica.

Al estudiar los rasgos de la personalidad de los adolescentes que abusan del alcohol, Llopis Llacer (1988) llegó a conclusiones similares al comprobar que los rasgos comunes más significativos eran la extroversión, nerviosismo y falta de control. A su vez Alterman (1990) estableció la relación significativa entre desinhibición y propensión al consumo de alcohol. Comprobó además que aquellos adolescentes que habían tenido una infancia conflictiva y antisocial, desarrollaban alcoholismo en una proporción significativamente más elevada. Windle y Well (1991) obtuvieron similares resultados afirmando que la conducta infantil antisocial es un factor de riesgo significativo para la conducta de ingesta en la adolescencia.

En un estudio reciente realizado por Murphy en la Universidad de Maryland (2001) con 303 parejas de las cuales el hombre era alcohólico, se constató que los hombres alcohólicos que ejercían violencia doméstica se caracterizaban por una personalidad antisocial característica y problemas de relación. Murphy encontró una relación significativa entre alcoholismo y conducta antisocial aun controlando todas las demás variables.

\section{El medio ecológico}

En este ámbito es preciso distinguir, por una parte, los factores predisponentes, que son la base de estudios epidemiológicos, como el sexo, edad de inicio, status socioeconómico, cultura, etc., y por otra parte, los agentes de socialización que centran mayor atención en las atribuciones causales o variables condicionantes del inicio y prevalencia del consumo de alcohol en la adolescencia.

\section{La presión del medio}

En un estudio sobre las circunstancias concomitantes con el inicio en la ingesta de alcohol, Cárdenas (1987) encontró que los medios de comunicación habían sido determinantes del inicio en el consumo y, entre ellos, atribuye gran influencia a la publicidad en la adquisición del hábito de consumo de alcohol en la adolescencia ya que el sujeto recibe un conjunto de mensajes con expectativas, en relación con la ingesta de alcohol, que son integradas por el sujeto y favorecen la ingesta porque suponen una serie de consideraciones positivas hacia el alcohol. Weiss en la década de los noventa estudió ampliamente este tema y concluye que el medio ecológico y la cultura del propio adolescente condicionan la percepción de los mensajes publicitarios y también el grado de influencia de los mismos.

Vega (1998) afirma que los medios de comunicación desempeñan un papel ambigüo, pues mientras, por una parte, muestran con todo dramatismo los efectos del consumo abusivo de alcohol entre la población más joven, sirven, al mismo tiempo, de soporte para todo tipo de bebidas alcohólicas, con una publicidad específica dirigida precisamente a esta población. 
Sin embargo, no se le puede imputar a la publicidad la responsabilidad de la prevalencia e incidencia del consumo de alcohol, porque en ese caso no tendría explicación la existencia de jóvenes abstemios que están igualmente expuestos al peligro de los mensajes publicitarios. La creencia de que los jóvenes pueden ser manipulados simplemente con sugerencias en los medios de comunicación es simple y errónea. Es necesario conocer el medio ecológico del joven para entender el impacto de la publicidad y lo que significa para él un determinado mensaje y el porqué de su respuesta.

En la actualidad, en España, la vigente legislación sobre el control de los mensajes publicitarios restringe la publicidad sobre bebidas alcohólicas, pero a través de los medios de comunicación recibimos permanentemente mensajes claros o subliminales sobre las ventajas y beneficios de consumir bebidas alcohólicas e, incluso, del abuso de las mismas. Dado que no existe una educación sistemática sobre sus efectos y que el consumo en los menores no está sometido a un control real y eficaz, el impacto publicitario encuentra un terreno abonado para recibir el mensaje, porque la publicidad, como la droga, nos vende de contrabando aquello que en las condiciones socioexistenciales actuales de vida se nos niega. La publicidad como la droga nos devuelve el sueño de la felicidad» (Younis, 1993).

Pero la presión del medio no es ejercida solamente desde la publicidad, sino que tiene mucha más influencia la presión del entorno en el que se desenvuelve el sujeto, ya que hay ambientes en los que la ingesta de bebidas alcohólicas está considerada como un hábito indispensable para relacionarse socialmente. En determinados trabajos parece imprescindible tener una copa en la mano para desempeñarlos. A este respecto Hemmingssons (2001) estudió las características de esta población y encontró que los jóvenes que ya abusaban del alcohol al desempeñar un trabajo de esas caracteríticas, inevitablemente desemboca en el alcoholismo.

\section{El grupo de iguales}

El grupo de iguales es un agente de socialización muy importante, sobre todo en la adolescencia, por el fuerte modelado que ejerce en las actitudes de los miembros del grupo. El joven busca la aprobación e integración en el grupo y para ello adopta las actitudes y conductas predominantes en el grupo e impuestas a cada miembro para su aceptación. De ahí que en la tipología del adolescente que consume bebidas alcohólicas, un rasgo predominante y común sea su fuerte dependencia del grupo (Calafat, 1986). El consumo de alcohol en la adolescencia forma parte del aprendizaje social. Cuando el grupo de iguales bebe alcohol, el adolescente necesita buscar su aprobación compartiendo la conducta de ingesta. Por ello, la mayor influencia le viene de su grupo de iguales, con quienes aprende y comparte sus primeras experiencias con el alcohol. El consumo del adolescente aumenta bajo la presión de la necesidad afiliativa (Smith, 1989).

Guerrero (1990) estudió a adolescentes que abusaban del alcohol y destaca que la razón más importante para beber era la de «estrechar amistades en su grupo». Los grupos de iguales se rigen por unos determinados patrones. Los juegos de beber de los adolescentes miembros de un grupo se rigen por reglas y juegan un importante rol de socialización; sirven para definir el status de cada miembro dentro del grupo, ya que las reglas marcan que cada miembro pueda beber más, pero nunca menos que sus compañeros (Pedersen, 1990). 
El consumo se inicia como un juego (el botellón, por ejemplo) que es importante para muchos jóvenes porque supone la aceptación e integración en un grupo de iguales. Los juegos proporcionan una estructura organizada de interacción que se caracteriza por unas fuertes relaciones de interdependencia entre los miembros del grupo y los participantes toman parte en ese juego con intención de beber (León Fuentes, 1990). Pedersen considera que el joven cuando participa en este juego de beber, se somete a las normas del grupo y pierde el control de su conducta. Generalmente el joven que consume bebidas alcohólicas manifiesta que todos o la mayoría de sus amigos ingieren bebidas alcohólicas habitualmente, y argumenta que lo hace para divertirse o para ser como los demás de su grupo (Repetto y Senra, 1997), razones ambas de gran peso en la conducta social del adolescente, por lo que se puede considerar al grupo de amigos con los que se relaciona el adolescente como un factor predictivo de su conducta de ingesta. A similares conclusiones llega Ariza Cardenal (2000) en un estudio con 2140 escolares barceloneses, manifestando que el grupo de iguales es el más importante factor predictivo de la conducta de ingesta problemática en un adolescente.

\section{La educación}

El estudio de los factores sociopedagógicos ha generado una nutrida y fructífera corriente de investigación en los últimos años, motivada en parte porque el consumo de alcohol se ha puesto de moda entre los adolescentes y cada vez es más precoz la edad de inicio. Aunque parece unánimemente admitido que los factores sociopedagógicos no se pueden considerar determinantes causales del consumo de alcohol, si es generalizado el criterio de que la probabilidad de que aparezcan problemas con el alcohol aumenta cuando, a los factores personales de tipo biológico o psicológico, se le añaden condiciones sociales desfavorables, y no solamente referidas al status social de los padres, sino al complicado ambiente social de cada joven, es decir, el hogar paterno, la escuela y su círculo de amigos o conocidos.

Las investigaciones de los factores educacionales, con una u otra orientación, se basan en el estudio de la influencia de las actitudes y la formación de la estructura actitudinal como base de la conducta. Las actitudes de una persona definen y conforman su personal visión del mundo y su característica forma de pensar. De ahí el gran interés que ha despertado el estudio de las actitudes del adolescente hacia el alcohol por su estrecha relación dinámica con la conducta manifiesta (Davies, 1983).

Dado que la conducta de consumo de bebidas alcohólicas forma parte de la cultura y de la conducta social del adulto, Maddox (1970) afirma que el adolescente aprende la conducta bebedora y la integra en todo su proceso de aprendizaje hacia la adultez. En este proceso tiene una gran importancia el aprendizaje de los roles sociales.

La educación para la salud ha sido sugerida por muchos investigadores como una necesidad en la etapa escolar (Cruzado, 1994), aunque no parece suficiente con la educación para la salud, sino que los programas de prevención deben dirigirse desde una edad temprana al desarrollo de habilidades sociales y al entrenamiento en la aceptación de normas sociales con el fin de reducir la motivación para iniciar el consumo de alcohol (Donaldson, 1994).

Como ya mencionamos anteriormente Kandel llegó a la conclusión de que el consumo y abuso del consumo de alcohol en la infancia y adolescencia sólo se puede explicar como 
una función de diversos factores, pero sus conclusiones se inclinan por una explicación pedagógica en la que dos procesos explican la conducta de ingesta:

1. La imitación, mediante la cual los jóvenes modelan su conducta y actitudes por simple observación de la conducta de otras personas significativas para ellos.

2. El refuerzo social. Los adolescentes internalizan y desarrollan los hábitos, conductas y valores aprobados por esas personas significativas para ellos».

Es precisamente la falta de información sobre los efectos del alcohol uno de los factores causales más importantes del inicio y prevalencia de la conducta de ingesta en la adolescencia (Repetto y Senra, 1997), alcanzando porcentajes elevados (73\%) la población jóvenes que abusan del alcohol y carecen de información sobre sus efectos, estando indefensos ante los riesgos del abuso. Kadden (2001) estudió las actitudes y conocimientos de una muestra de jóvenes hacia las bebidas alcohólicas y, a partir de los resultados obtenidos, realizó una campaña conductual-cognitiva con aquellos jóvenes que abusaban del alcohol, obteniendo unos resultados que le permitieron afirmar que las estrategias cognitivas son fundamentales para educar al joven en el consumo responsable.

Numerosos investigadores aportan sus experiencias con diferentes enfoques que evidencian la gran importancia de la educación: Glider realizó un estudio en el campus de Arizona (2001) a partir del cual desarrolló una campaña preventiva basada en mensajes sociales normativos, cuyos resultados le permiten concluir que la falta de normas sociales contribuye a que el joven desarrolle una conducta antisocial de ingesta.

Para Peleg (2001) la carencia de habilidades sociales es un factor que propicia el abuso de bebidas alcohólicas, en muchas ocasiones como recurso sustitutivo de las mismas; de hecho, tras la aplicación de un programa basado en la teoría de habilidades sociales de Botvin, comprobó, en un seguimiento posterior de dos años, que el grupo experimental había reducido el consumo de bebidas alcohólicas.

\section{A modo de reflexión}

Del análisis y revisión de los distintos enfoques que estudian la etiología del abuso de alcohol en la adolescencia, podemos deducir que su estudio y prevención solo puede abordarse teniendo en cuenta los múltiples factores que intervienen en su causalidad y partiendo del medio ecológico concreto para elaborar un programa educativo que abarque todos los factores intervinientes (Senra, 2000).

Parece evidente que no basta con puntuales campañas preventivas para adolescentes, cuando ya, por el mero hecho de serlo, constituyen una población de riesgo, ya que las estadísticas reflejan que un alto porcentaje se inicia en el consumo en edades muy tempranas. La educación, dentro del marco institucional, se presenta como la alternativa que puede abordar desde la infancia la conformación de la estructura actitudinal del individuo, desarrollando su capacidad crítica para la toma de decisiones responsables. Por otra parte, la educación y entrenamiento en asertividad, dotarán al adolescente de recursos y habilidades para hacer frente a situaciones críticas y desenvolverse en diversos ambientes.

Si en la actualidad nadie pone en duda que la educación debe ser un proceso integral e integrador que abarque todas las dimensiones del hombre, apuntamos la gran importancia del papel que juega como proceso preventivo desde edades tempranas para sentar una sóli- 
da base de pensamiento constructivo sobre la que edificar un engranaje actitudinal positivo que le permita compensar las carencias familiares y afrontar su proyecto de vida.

\section{Referencias bibliográficas}

Alonso, F. (1988): Alcoholdependencia. Personalidad del Alcohólico. Barcelona, Edit. Salvat, S.A.

Alterman, A. I. y Tarter, R. E. (1986): The transmision of psychological vulnerability implication of alcohol. Etiology. Journal Nerv. Mental disorders, vol. 171, 147-154.

Alterman, A. I. \& cols. (1990): Heavy drinking and its correlates in young men. Addictive behaviors, vol. $15,95-103$.

Ariza, C. \& Nebot, M. (2000): Factores asociados con el consumo problemático de alcohol en escolares. J. Adolesc. Health, Vol. $27\left(\mathrm{n}^{\circ}\right.$ 6) 425-433.

Barnes, G. M. (1990): Prediction of adult's drinking patterns from the drinking of their parents. Journal Stud. on Alcohol, Vol. 51 ( $\mathrm{n}^{\circ}$ 6) 523-27.

Black, N. M. (1994): Drug use, drug trafficking, and weapon carrying amon low-income, AfricanAmerican, early adolescents boys. Pediatrics, Vol. 93 (nº 6) 1065-72

Blankfield, A. (1991): Women alcohol dependence and crime. Drug alcohol dependence, vol. 27 $\left(n^{\circ} 2\right)$ 185-190.

Cadoret, R. (1980): Development of the alcoholism in adoptees raised apart from alcoholic biological parents. Archs. Gen. Psychiatry, Vol. 37, 561-63.

Calafat, A. y cols. (1986): Tipología de los consumidores y no consumidores de alcohol en Enseñanza Media. Drogalcohol, Vol. XI (n 1) 3-15

Cárdenas, C. y Moreno, B. (1987): La ingesta de alcohol en la adolescencia. Revista Española de Drogodependencias, vol. 12 ( $\mathrm{n}^{\circ}$ 4), 243-55

Corrao, G. (1995): Alcohol-related problems within the family and global functioning of the children: a population-based study. Soc. Psychiatry Psychiatr Epidemiol Vol. 28 (n ${ }^{\circ}$ 6) 304-8

Cruzado, J. (1994): Consumo de alcohol entre escolares de $7^{\circ}$ de EGB. Atención Primaria, Vol. 13 $\left(n^{\circ} 9\right)$ 495-97.

Davies, J. B. (1982): The transmission of alcohol problems in the family. Londres, Oxford Croom Helm.

Davies, J. B. (1983): Children's and adolescent's attitudes towards alcohol and alcohol dependence. En O. Jeaneret Child Health and development, vol. 2, 27-42 Geneve.

Donaldson, D. I. (1994): Testing the generalizability of intervening mechanism theories: understanding the effects of adolescent drug use prevention interventions. Journal Behavior Med. Vol. 17 $\left(n^{\circ} 2\right) 195-216$.

Eysenck, H. J. (1965): The causes and cures of neurosis. London, Rutledge and Kegan.

Glider, P. \& cols. (2001): Challenging the collegiate rite of passage: a campus-wide social marketing media. J. Drug Education, Vol. 31 (nº 2) 207-220

Goodwin, D.W. et als. (1973): Alcohol problems in adoptees raised apart from alcoholic biological parents. Arch. Gen. Psychiatric, vol. 28, 238-243.

Goodwin, D.W. (1979): Genetics determinants of alcoholism. Págs. 59-82. New York, Mc Graw Hill.

Griffin-Shelley, E. (1990): Sex role perceptions in chemical dependent subjects: Adult versus adolescents. Int. J. Addictive, vol. 25 ( $\left.\mathrm{n}^{\circ} 12\right)$ 1383-91. 
Guerrero, C. y otros (1990): El método el informante para conocer la percepción de hábitos de consumo de jóvenes de 15-19 años. Revista Española de Drogodependencias, vol. 15 (n 2) 79-96.

Hemmingssons, T. (2001): Development of alcoholism: interaction between heavy adolescent drinking and later low sense of control over work. Alcohol alcohol, Vol. 36 (n 3), 207-212.

Hyphantis, T. (1991): Alcohol and drug use, family situation and school performance in adolescents children of alcoholics. Internal J. Soc. Psychiatry, vol. 37 (nº 1) 35-42.

Ibáñez, P. y Senra, M. (1999): Atribuciones causales de la autoestima en los hijos de alcohólicos. Evaluación e Intervención Psicoeducativa, Vol. I (nº 2-3) 211-222.

Jacob, T. (1989): Alcoholism and family interaction: an experimental paradigm. Am. J. Drug Alcohol Abuse, Vol. 15 ( $\mathrm{n}^{\circ}$ 1), 73-91.

Jellineck, E. M. (1960): The disease concept of alcoholism. Conn. New Haven, College \& University Press.

Kaij, L. (1960): Alcoholism in twins: Studies on the etiology and sequels of abuse of alcohol. Stockholm, Almquist \& Wiksell International.

Kadden, R. M. (2001): Behavioral and cognitive-behavioral treatments for alcoholism: research opportunities. Addict. Behavior, Vol. 26 ( ${ }^{\circ}$ 4) 489-507.

Kandel, B. (1983): Socialization and adolescent drinking. 66-75. New York. O. Janeret, Karger.

Killerman, M. (1990): Alcohol-responsive myoclonic dystonia in a large family: inheritance and fhenotyp. Mov. Dosord., vol. 5 ( $\mathrm{n}^{\circ}$ 4) 270-79.

Kornblit, A. L. (1990): Recaídas en drogadicción. Factores de riesgo. Revista Española de Drogodependencias, vol. 15 ( $\mathrm{n}^{\circ}$ 2) 97-109.

León, J. L. (1990): Reflexiones sobre la situación actual del consumo de alcohol y drogas por la juventud. Revista Española de Drogodependencias, vol. 15 (n 1) 45-51

Lex, B. W. (1991): Some gender differences in alcohol and polysubstance users. Health Psychology, vol. $10\left(n^{\circ} 2\right) 121-132$.

Llopis, J. y cols. (1988): Circunstancias concomitantes con la edad de inicio en el consumo en una muestra de alcohólicos crónicos. Revista Española de Drogodependencias, vol. 13 (n 2) 93-107.

Maddox, G. L. (1970): The domestical drugs drinking among collegions. en Adolescence and alcohol, Cap. 2, 33-47. New Haven. College \& University Press.

Molina, B. S. (1994): A comparison of mechanisms undrlying substance use for early adolescent children of alcoholics and controls. J. Study Alcohol Vol. 55 (n 3) 269-75

Murphy, C. M. (2001): Correlates of intimate partner violence among male alcoholic patients. J. Consult. Clin. Psychol., vol. 69 (nº 3) 528-540.

Natera Rey, G. y otros (2001): Influencia de la historia familiar de consumo de alcohol en hombres y mujeres. Salud Pública Mexicana, vol. 43 (n 1) 17-26.

Pandina, R. J. \& Johnson, V. (1989): Familial drinking history as a predictor of alcohol and drug consumption among children. Journal of Studies on Alcohol, vol. 50 (n 3 ) 245-53.

Pedersen, W. (1990): Drinking games adolescents play. British J. of Adiction, vol. 85 (n 11) 1483-90.

Peleg, A. \& cols. (2001): Outcomes of a brief alcohol abuse prevention program for Israeli high school students. J. Adolescenc Health, Vol. 28 (n 4) 263-69

Penik, E. C. \& cols. (1990): A comparison of familial and nonfamilial male alcoholic patients without a psychological disorders. J. Stud. Alcohol. vol. 51 (n $\left.{ }^{\circ}\right)$ 443-47.

Power, C. (1990): The role of the family formation and disolution in shaping drink behavior in early adulthood. British J. of Addiction, vol. 85, 521-30 
Rearden, J. J. (1989: Self concept and drinking problems of college students raised in alcoholic abused homes. Addictive behaviors, vol. 14, 225-227.

Repetto, E. y Senra, M. (1997): Análisis de algunos factores educativos en el consumo de alcohol en la adolescencia. Revista de Investigación Educativa, vol. 15 (nº 1) 31-42

Roe, A. (1944): The adult adjustment of children of alcoholic parents raised in a foster-homes. Q.J. Studies on alcohol. Vol. 5, 378-93.

Ross, L. T. \& Hill, E. M. (2001): Drinking and parental unpredictability among adult children of alcoholics: a pilot study. Subst. Use Misuse, Vol. 36 (nº 5) 609-638.

Santo domingo, J. y Rodríguez,, B. (1989): Evolución de los hábitos de consumo de alcohol y de la dependencia alcohólica en España. Revista Española de Drogodependencias, vol. 14 ( $\left.\mathrm{n}^{\mathrm{o}} 3\right)$ 167-75.

Schuckit, M. A. (1984): Subjective responses to alcohol in sons of alcoholics and control subjects. Arch. Gen. Psychiatry, Vol. 41, 879-884.

Senra, M. (2000): Relación del clima familiar con el hábito de consumo de alcohol de los hijos de alcohólicos. Revista de Ciencias de la Educación n 181, 81-89.

Senra, M. (2000). La intervención psicopedagógica para la prevención del alcoholismo juvenil. Studia Académica, $\mathrm{n}^{\circ}$ 10, 175-198.

Stabenau, J. R. (1990): Additive independent factors to predict risk for alcoholism. Journal of studies on alcohol, vol. 51 ( $\left.\mathrm{n}^{\circ} 2\right)$ 164-74.

Tambs, K. (1990): Alcohol consumption in parents and offspring: a study of the family structure. Acta Psychiatr. Scand., vol. 82 ( $\mathrm{n}^{\circ}$ 2) 145-51.

Turner, A. P. (2000): Family risk factors for alcohol-related consequences and poor adjustment in fraternity and sororoty members: exploring the role of parent-child conflict. Journal Studies on Alchol, vol. 61 ( $\mathrm{n}^{\circ}$ 6) 818-826.

Tweed, S. H. (1991): Adult children of alcoholics: profiles of wellness amidts distress. J. Stud. Alcohol, vol. $52\left(\mathrm{n}^{\circ} 2\right)$ 133-41.

Vallejo, J. A. (1981): Introducción a la Psiquiatría.Barcelona, Edit. Científico Médica.

Velleman, R. \& Orford, J. (1990): TYoung adult offspring of parents with drinking problems: its inmediate effects. British journal Clin. Psychology, vol. 29 (n $\mathrm{n}^{\mathrm{O}}$ 3) 297-317.

Vollicer, L. (1984): Relationship of family history of alcoholism to patterns of drinking and physiological dependence. Drug alcohol dependence, vol. 13, 215-223.

Windle, M. (1991): The difficult temperament in adolescents: Associations with substance abuse and family support. J. Clin. Psychology, vol. 47 (n 2) 310-15.

Witold, T. \& cols. (1989): The role of the family in alcohol education and alcohol abuse in Poland. Medicine and Law, vol. 8, 267-73. 